\title{
Subcarrier and Power Allocation for LDS-OFDM System
}

\author{
Mohammed AL-Imari*, Muhammad Ali Imran*, Rahim Tafazolli*, and Dageng Chen ${ }^{\dagger}$ \\ ${ }^{*}$ Centre for Communication Systems Research,University of Surrey, Guildford GU2 7XH, Surrey, U.K. \\ Email: \{M.Al-imari,M.Imran,R.Tafazolli\}@ surrey.ac.uk \\ ${ }^{\dagger}$ Huawei Technologies Co., Ltd. No.2222, Xin JinQiao Road, Shanghai, China, 201206 \\ Email: Dagengchen@huawei.com
}

\begin{abstract}
Low Density Signature-Orthogonal Frequency Division Multiplexing (LDS-OFDM) has been introduced recently as an efficient multiple access technique. In this paper, we focus on the subcarrier and power allocation scheme for uplink LDSOFDM system. Since the resource allocation problem is not convex due to the discrete nature of subcarrier allocation, the complexity of finding the optimal solutions is extremely high. We propose a heuristic subcarrier and power allocation algorithm to maximize the weighted sum-rate. The simulation results show that the proposed algorithm can significantly increase the spectral efficiency of the system. Furthermore, it is shown that LDSOFDM system can achieve an outage probability much less than that for OFDMA system.
\end{abstract}

Index Terms-LDS-OFDM, radio resource management, fairness, multiuser diversity.

\section{INTRODUCTION}

Multicarrier transmission has been considered as a promising technique for high data rate transmission in wireless and wired applications. Orthogonal Frequency Division Multiplexing (OFDM) is one of the prominent multicarrier transmission techniques. For wireless applications, an OFDM-based system offers greater immunity to multipath fading and impulsive noise with low complexity per subcarrier equalization [1].

Orthogonal Frequency Division Multiple Access (OFDMA) is an efficient extension of OFDM transmission to a multiuser communication scenario. In OFDMA systems, the set of subcarriers is divided into several mutually exclusive subsets that are assigned to different users for simultaneous transmission.

Low Density Signature OFDM (LDS-OFDM) technique is introduced in [2] as an efficient uplink multicarrier multiple access scheme. The technique combines the benefits of OFDM based multicarrier transmission with a recent idea on LDS based spreading proposed for Code Division Multiple Access (CDMA) systems in [3]. LDS-OFDM combines the two concepts of LDS and OFDM to establish an efficient multiple access over wideband channels. In LDS-OFDM, due to low density signature structure, every data symbol will only be spread on a small subset of subcarriers (effective processing gain), and also every subcarrier will only be used by a small subset of data symbols that could belong to different users.

The LDS-OFDM technique is able to exploit both the channel and the multiple access interference diversities over the frequency domain. In [2] it is shown that LDS-OFDM outperforms the performance of OFDMA over a typical multipath fading channel and under different spectral efficiency conditions. On the other hand, LDS-OFDM detector has larger complexity than conventional single-user receivers. The incurred computational complexity is justified thanks to improved performance and also due to the lesser complexity restrictions of the network side where the LDS-OFDM receiver is employed.

In multiuser systems, the signal of each user experiences independent channel realizations. Therefore, the radio resources (subcarrier and power) should be dynamically allocated to the users to utilize the multiuser diversity [4]. There have been a lot of research works considering the radio resource management in the OFDMA system (e.g. [5]-[7]). The subcarrier and power allocation for LDS-OFDM system has not explored yet. In the previous work fixed resource allocation is considered for LDS-OFDM system. The main drawback of fixed resource allocation is that multiuser diversity is not been exploited. There is plenty of room to exploit the high degree of flexibility of radio resource management in the context of LDS-OFDM system. As the channel frequency responses are different at different frequencies or for different users, dynamic subcarrier and power allocation can significantly improve the performance of LDS-OFDM system. Furthermore, in LDSOFDM, there is no exclusivity in the subcarrier allocation and several users can share a subcarrier if feasible.

In this paper, we consider the subcarrier and power allocation problem for LDS-OFDM system. The objective is to maximize the system throughput and ensure fairness among the users. We propose a heuristic subcarrier and power allocation algorithm to maximize the weighted sum-rate. By following the guidelines gained from the power allocation for generic multicarrier MAC, we adopt a greedy approach in the proposed algorithm. We show that the proposed algorithm can enhance the system performance comparing to the fixed resource allocation scheme.

This paper is organized as follows: Section II presents the LDS-OFDM system model. The formulation for the weighted sum-rate maximization is provided in section III. In section IV, we propose a heuristic subcarrier and power allocation algorithm. In section $\mathrm{V}$, we evaluate the performance of the proposed algorithm through Monte Carlo simulations. Finally, section VI is devoted to concluding remarks. 


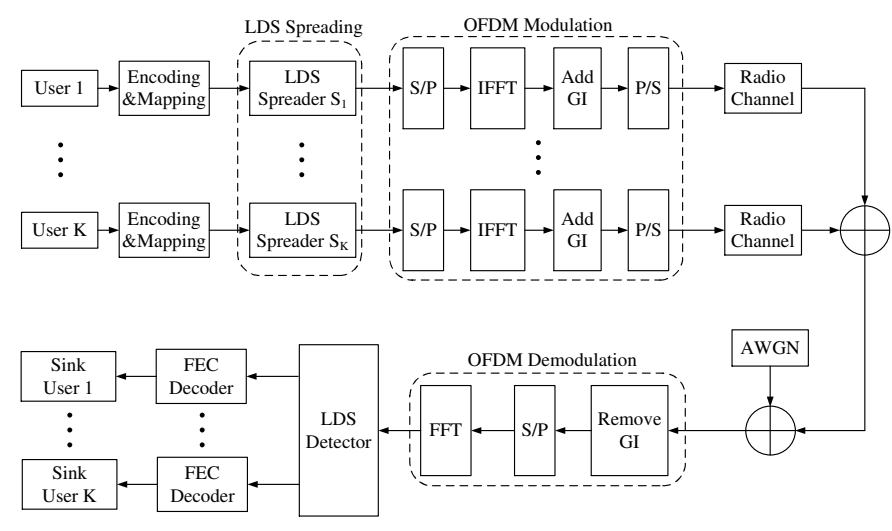

Fig. 1. LDS-OFDM block diagram.

\section{LDS-OFDM SYSTEM MODEL}

In this section, a single cell uplink LDS-OFDM system model is presented. The key notations are listed in Table I. Consider an uplink LDS-OFDM system with a set of users $\mathcal{K}=\{1, \cdots, K\}$ transmitting to the same base station where the base station and each user are equipped with a single antenna. The total frequency band is divided into a set of subchannels (subcarriers/tones) $\mathcal{N}=\{1, \cdots, N\}$. The frequency response is assumed flat within each subcarrier, i.e. the intersymbol interference is completely removed by exploiting the OFDM technique. A user $k \in \mathcal{K}$ can transmit over a subset of the subcarriers, with transmission power $p_{k, n}$ over subcarrier $n \in \mathcal{N}$ subject to individual maximum power constraints $P_{k}: \sum_{n \in \mathcal{N}} p_{k, n} \leq P_{k}$.

The conceptual block diagram of an uplink LDS-OFDM system is shown in Fig. 1. In an LDS-OFDM system, the signal spreading is performed in the frequency domain. The data streams are first multiplied with the low density spreading signatures and then transmitted over different subcarriers. Each data symbol is spread using a low density signature. The low density signature is a sparse vector consisting of $N$ chips. Among the $N$ chips only $d_{v}$ chips have non-zero values, where $d_{v}$ is the effective spreading factor. The positions (indices) of the non-zero chips represent the subcarrier mapping (allocation). Each user's generated chip will be transmitted over a subcarrier of the OFDM system.

As users are not bound to exclusively use the subcarriers, at the receiver side users' signals that are using the same subcarrier will be superimposed. However, the number of users interfere in each subcarrier is much less than the total number of users, $d_{c} \ll K$, where $d_{c}$ denotes the number of users interfere in each subcarrier. The LDS structure can be captured by a low density graph, thus the detection of LDS-OFDM can be done using close to optimum multiuser detection based on Message Passing Algorithm (MPA) presented in [3]. The complexity of the multiuser detection for LDS-OFDM will turn out to be $\mathcal{O}\left(|\mathbb{X}|^{d_{c}}\right)$ which is significantly reduced comparing to complexity of order $\mathcal{O}\left(|\mathbb{X}|^{K}\right)$ for optimal multiuser detection [3], where $\mathbb{X}$ denotes the constellation alphabet.
TABLE I

NOTATIONS.

\begin{tabular}{|c|c|}
\hline Notation & Physical Meaning \\
\hline$N$ & Total number of subcarriers \\
\hline $\mathcal{N}$ & Set of all subcarriers \\
\hline$n$ & Subcarrier index \\
\hline$K$ & Total number of users \\
\hline $\mathcal{K}$ & Set of all users \\
\hline$k$ & User index \\
\hline$P_{k}$ & Maximum transmit power for user $k$ \\
\hline$w_{k}$ & Weight associated to user $k$ \\
\hline
\end{tabular}

More details regarding the LDS receiver and the LDS-OFDM system can be found in [2], [3].

\section{PRoblem Formulation}

We will consider the problem of maximizing the sum-rate with fairness consideration for LDS-OFDM system. The base station periodically estimates the uplink channel states of all subcarriers for each user. Based on the obtained channel state information, the base station then assigns the subcarriers and power to each user through a reliable signaling channel. Using the sum-rate as the optimization criteria is sometimes unfair to the cell edge users. Therefore, it is essential to have a trade-off between the spectral efficiency and fairness among the users in the radio resource allocation schemes [8]. So, we will consider weighted sum-rate maximization.

As pointed out before, in LDS-OFDM system the users' signals are overlapped at the receiver, and joint processing is implemented to separate the users' data. In order to formulate the resource allocation problem for LDS-OFDM system, we will assume that there is successive decoding at the receiver in order to perform the joint processing. The decoding order can be represented by a permutation $\pi$, and the users are decoded in the order $\pi(1), \pi(2), \cdots, \pi(K)$. Thus, the user who will be decoded first, $\pi(1)$, will see interference from all the other users $\pi(2), \cdots, \pi(K)$, and the second user to be decoded will see interference from the users $\pi(3), \cdots, \pi(K)$, and so on. Accordingly, the set of users that interfere to the $k$ th user can be represented by:

$$
\mathcal{I}_{k}=\left\{\pi(i): i>\pi^{-1}(k)\right\} .
$$

Let $x_{k, n}$ be the subcarrier allocation index, where $x_{k, n}$ equal to 1 if $p_{k, n}>0$, and 0 if $p_{k, n}=0$. The users are assigned weights, as a fairness mechanism, where users with bad channel conditions will be assigned higher weights. Consequently, the weighted sum-rate maximization problem can be formulated as follows:

$$
\max _{x_{k, n}, p_{k, n}} \sum_{k \in \mathcal{K}} w_{k} \sum_{n \in \mathcal{N}} x_{k, n} \log \left(1+\frac{g_{k, n} p_{k, n}}{\sum_{i \in \mathcal{I}_{k}} x_{i, n} g_{i, n} p_{i, n}+N_{0} B_{N}}\right)
$$


subject to

$$
\begin{gathered}
\sum_{k \in \mathcal{K}} x_{k, n} \leq d_{c}^{\star}, \quad \forall n \in \mathcal{N} \\
\sum_{n \in \mathcal{N}} p_{k, n} \leq P_{k}, \quad \forall k \in \mathcal{K} \\
p_{k, n} \geq 0, \quad \forall k \in \mathcal{K}, \quad n \in \mathcal{N} \\
x_{k, n} \in\{0,1\}, \quad \forall k \in \mathcal{K}, \quad n \in \mathcal{N}
\end{gathered}
$$

Here, $g_{k, n}$ is the channel gain that user $k$ experiences on subcarrier $n . N_{0}$ and $B_{N}$ are the noise power spectral density and the subchannel bandwidth, respectively. The maximum number of users per subcarrier is represented by $d_{c}^{\star}$, and $w_{k}$ is the weight associated to user $k$. Constraint (4) provides per user transmission power limitation whereas constraint (3) imposes the receiver's constraint on the number of users per subcarrier. The above problem is a combinatorial one due to the binary variable $x_{k, n}$, which is intractable for a large system [9].

\section{Proposed Algorithm}

We will propose a heuristic recourse allocation algorithm for LDS-OFDM system based on the insights gained from the optimal power allocation for generic multicarrier MAC [4]. For the generic multicarrier MAC, all the users can transmit on all the subcarriers subject to an individual maximum power constraints. In [4], the optimal power allocation to maximize the weighted sum-rate for the generic multicarrier MAC has been derived. The solution contains various steps, which we summarize as follows:

- Let $N_{0}+z$ denotes the current "interference level" due to the background noise and received power of users not cancelled yet. Starting at received power $z=0$, the optimal solution is obtained by adding a user, at each interference level, which will lead to the largest positive marginal increase in the objective function.

- The optimal solution is achieved by successive decoding, and the decoding order is in increasing order of the users' weights $w_{\pi(1)} \leq w_{\pi(2)} \cdots \leq w_{\pi(K)}$.

The optimal solution for generic multicarrier MAC provides guidelines for us to design our algorithm for subcarrier and power allocation in LDS-OFDM system. Firstly, the optimal power allocation for generic multicarrier MAC is achieved by a greedy approach. A particular user will be allocated power on a given subcarrier if it results in the maximum increase in the objective function at a given interference level. Secondly, the decoding order is in increasing order of the users' weights.

Inspired by these two aspects reflecting the greedy approach, we propose a heuristic subcarrier and power allocation algorithm for LDS-OFDM system. Here, each user will experience interference only from the users with higher weights, and the set of users that interfere to the $k$ th user can be defined as:

$$
\mathcal{I}_{k}=\left\{i: w_{i}>w_{k}\right\} \text {. }
$$

Let us define the utility of the $k$ th user on subcarrier $n$ as follows:

$$
u_{k, n}=w_{k} \log \left(1+\frac{g_{k, n} p_{k, n}}{\sum_{i \in \mathcal{I}_{k}} x_{i, n} g_{i, n} p_{i, n}+N_{0} B_{N}}\right) .
$$

The algorithm iteratively allocates the subcarriers one by one. For each subcarrier $n$ that has not been fully loaded (i.e. $\sum_{k \in \mathcal{K}} x_{k, n} \leq d_{c}^{\star}$ ), we allocate the subcarrier to the user who has the maximum non-zero utility value, $u_{k, n}$. The algorithm will stop when all the subcarriers are fully loaded or there is no user that has non-zero utility on unallocated subcarrier.

Let $\mathbf{J} \in \Re_{+}^{K \times N}$ be the interference matrix that indicates the interference every user will see on every subcarrier from the other users, where $j_{k, n}=\sum_{i \in \mathcal{I}_{k}} x_{i, n} g_{i, n} p_{i, n}$. Define $\mathcal{A}_{k}$ as the set of subcarriers allocated to user $k$, and $\mathcal{U}_{k}$ as the set of subcarriers that not allocated to user $k$ and allocated to less than $d_{c}^{\star}$ other users. Then the proposed subcarrier and power allocation algorithm is summarized as follows:

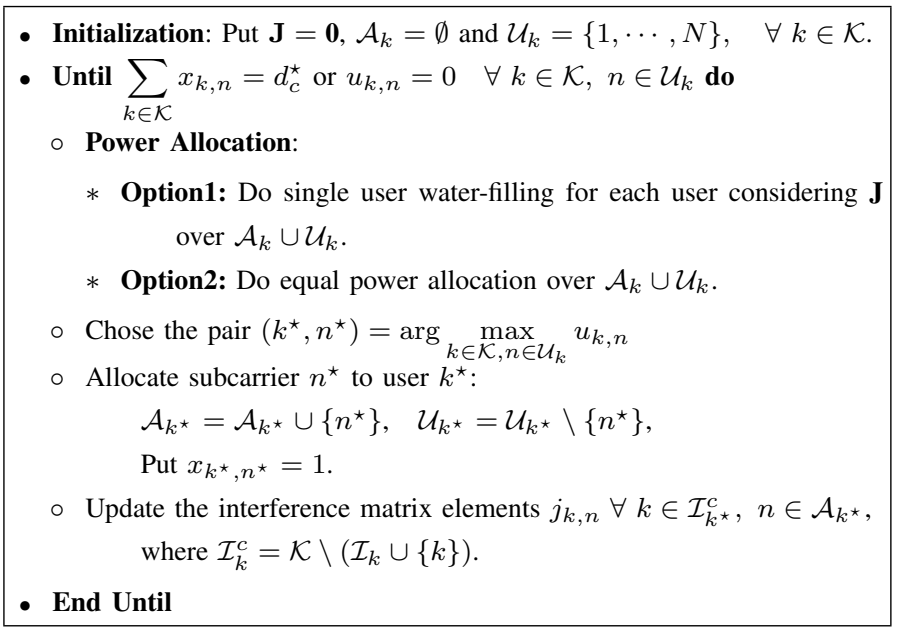

As the algorithm shows, the power allocation can be a waterfilling or equal power allocation. It is crucial to mention that the single user water-filling algorithm has taken into account the interference from the other users. The water-filling solution can be expressed as follows [10]:

$$
p_{k, n}=x_{k, n}\left[\nu_{k}-\frac{1}{j_{k, n}+N_{0} B_{N}}\right]^{+}
$$

where $[x]^{+}=\max (0, x)$ and $\nu_{k}$ is known as the water level that should satisfy the power constraint in (4).

\section{Simulation Results}

In this section, we evaluate the performance of LDSOFDM with subcarrier and power allocation using the proposed algorithm through Monte Carlo simulation. We will use the spectral efficiency and the outage probability as the performance metrics. The performance is compared with OFDMA system employing subcarrier and power allocation using the algorithm in [11]. However, we included 
users' weights in the algorithm for fairness and comparison purposes. The users' weights are calculated as the inverse of the users' path loses to ensure fairness among the users by giving high priority to users far from the base station (cell edge users). We consider a single base station with $1 \mathrm{~km}$ radius. In simulations, we assume that users' location is randomly generated and uniformly distributed over the cell. The maximum transmit power of each user is 1 Watt. The system bandwidth is $5 \mathrm{MHz}$ consisting of 32 subcarriers. The link gain between the base station and a user is given as the product of path loss, shadowing and fast fading effects. ITU pedestrian B is adopted for fast fading generation. The path loss is obtained by the modified Hata urban propagation model, which is given by (in $\mathrm{dB}$ ) [11]:

$$
\begin{cases}122+38 \log (d), & \text { if } d \geq 0.05 \mathrm{~km} \\ 122+38 \log (0.05), & \text { if } d<0.05 \mathrm{~km}\end{cases}
$$

where $d$ (in kilometres) is the distance between the base station and the user. Lognormal shadowing is considered with mean value 0 and standard deviation of $8 \mathrm{~dB}$. The noise power spectral density is assumed to be $-120 \mathrm{~dB}$. For LDS-OFDM system, the maximum number of users per subcarrier $d_{c}^{\star}$ is chosen to be 6 . We will compare following scenarios:

- LDS-OFDM-WF: LDS-OFDM system employing dynamic radio resource allocation using the proposed algorithm with water-filling power allocation.

- LDS-OFDM-EP: LDS-OFDM system employing dynamic radio resource allocation using the proposed algorithm with equal power allocation.

- LDS-OFDM-Static: LDS-OFDM system with fixed subcarrier and power allocation independent of the channel conditions. The subcarriers are equally distributed among the users, and each user distributes its power uniformly on the allocated subcarriers.

- OFDMA-WF: OFDMA system with greedy radio resource allocation using the algorithm proposed in [11] with water-filling power allocation.

- OFDMA-EP: OFDMA system with greedy radio resource allocation using the algorithm proposed in [11] with equal power allocation.

Fig. 2 shows the spectral efficiency versus the number of users for LDS-OFDM and OFDMA systems. It can be observed that more spectral efficiency is achieved as the number of users increased. The increase in spectral efficiency is due to the multiuser diversity and the increase in the sum of users' power. The results show that significant increase in the spectral efficiency is achieved by dynamic resource allocation comparing to the static scheme for LDS-OFDM system.

Furthermore, we can observe that the difference between using water-filling and equal power allocation in LDS-OFDM

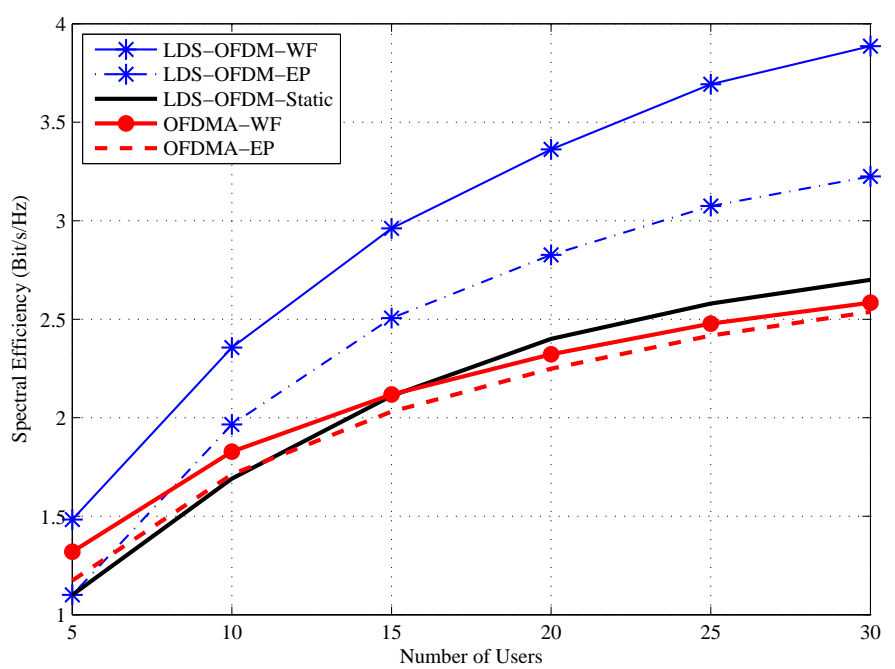

Fig. 2. Spectral Efficiency comparison for LDS-OFDM and OFDMA.

system is larger than in OFDMA system. As capacity is a logarithmic function of the Signal to Noise-plus-Interference Ratio (SNIR), the power allocation will have less effect in the high SNIR. In OFDMA system, there is no interference between the users, which results in high SNIR making the sum-rate less sensitive to the power allocation. On the other hand, in LDS-OFDM system the interference from the other users decreases the SNIR, which make the sum-rate more sensitive to the power allocation scheme. However, waterfilling requires higher signalling overhead, which will decrease the spectral efficiency gained.

Additionally, as it can be observed from the figure, LDSOFDM can achieve spectral efficiency higher than OFDMA. In OFDMA, subcarriers used by cell edge users (users with bad channels and high weights) are not available for users with high channel gain due to the exclusivity use of subcarriers. In LDS-OFDM, the subcarriers allocated to cell edge users can be used by the users with good channel conditions, which results in high spectral efficiency.

Table II shows the outage probability for all the simulated scenarios. If a user is not allocated any subcarrier the event is counted as an outage. The results show that the LDS-OFDMWF scenario performs the best among all the scenarios, from the outage probability perspective. LDS-OFDM with waterfilling has zero outage probability for all the cases, and all the users get access to the system for the cases under consideration. On the other hand, when the equal power allocation scheme is used for LDS-OFDM system, an outage occurs when the total number of users is increased. Nevertheless, the outage probability in LDS-OFDM system is much less than that in OFDMA system. As an example, for 30 users with an equal power allocation scheme, LDS-OFDM system achieves about 0.27 less outage probability comparing to OFDMA system.

Considering the results all together, it can be concluded that the dynamic subcarrier and power allocation can significantly 
TABLE II

Outage Probability.

\begin{tabular}{|c||c|c|c|c|c|c|}
\hline \multirow{2}{*}{\multicolumn{1}{|c||}{ Scenario }} & \multicolumn{7}{|c|}{ Number of Users } \\
\cline { 2 - 7 } & $\mathbf{5}$ & $\mathbf{1 0}$ & $\mathbf{1 5}$ & $\mathbf{2 0}$ & $\mathbf{2 5}$ & $\mathbf{3 0}$ \\
\hline LDS-OFDM-WF & 0 & 0 & 0 & 0 & 0 & 0 \\
\hline LDS-OFDM-EP & 0 & $9 \mathrm{e}-4$ & $7 \mathrm{e}-3$ & $25 \mathrm{e}-3$ & 0.05 & 0.09 \\
\hline OFDMA-WF & $27 \mathrm{e}-4$ & 0.06 & 0.15 & 0.22 & 0.3 & 0.36 \\
\hline OFDMA-EP & 0.02 & 0.1 & 0.18 & 0.25 & 0.32 & 0.37 \\
\hline
\end{tabular}

enhance the spectral efficiency of LDS-OFDM system comparing to the static scheme. Moreover, LDS-OFDM system can achieve a higher spectral efficiency and lower outage probability than that for OFDMA system.

\section{CONCLUSION}

Increasing interest in high data rate services demands high spectral efficiency techniques. In this regard LDS-OFDM was introduced as an efficient multiple access technique. In this paper, we considered the problem of joint subcarrier and power allocation for uplink LDS-OFDM system. The objective of the algorithm is to maximize the users' weighted sumrate. We proposed a greedy resource allocation based on the insights gained from the optimal power allocation for generic multicarrier MAC.

The simulation results showed that dynamic resource allocation can significantly increase the system spectral efficiency. It is shown that water-filling power allocation outperforms the equal power allocation in LDS-OFDM system. Furthermore, the results showed that LDS-OFDM system can achieve higher spectral efficiency comparing to OFDMA system. Also, LDSOFDM system can achieve an outage probability much less than that for OFDMA system. Future work will include further study and investigation to evaluate the performance of the LDS receiver under the proposed dynamic power and subcarrier allocation.

\section{ACKNOWLEDGMENT}

This work was supported by Huawei Technologies Co., Ltd, China.

\section{REFERENCES}

[1] J. Bingham, "Multicarrier modulation for data transmission: An idea whose time has come," IEEE Communications Magazine, vol. 28, no. 5, pp. 5-14, May 1990.

[2] R. Hoshyar, R. Razavi, and M. Al-Imari, "LDS-OFDM an efficient multiple access technique," in IEEE 71st Vehicular Technology Conference, May 2010, pp. 1-5.

[3] R. Hoshyar, F. Wathan, and R. Tafazolli, "Novel low-density signature for synchronous CDMA systems over AWGN channel," IEEE Transactions on Signal Processing, vol. 56, no. 4, pp. 1616-1626, April 2008.

[4] D. Tse and S. Hanly, "Multiaccess fading channels-Part I: Polymatroid structure, optimal resource allocation and throughput capacities," IEEE Transactions on Information Theory, vol. 44, no. 7, pp. 2796-2815, Nov. 1998.

[5] J. Jang and K. B. Lee, "Transmit power adaptation for multiuser OFDM systems," IEEE Journal on Selected Areas in Communications, vol. 21, no. 2, pp. 171-178, Feb. 2003.
[6] J. Huang, V. Subramanian, R. Agrawal, and R. Berry, "Joint scheduling and resource allocation in uplink OFDM systems for broadband wireless access networks," IEEE Journal on Selected Areas in Communications, vol. 27, no. 2, pp. 226-234, Feb. 2009.

[7] C. Y. Ng and C. W. Sung, "Low complexity subcarrier and power allocation for utility maximization in uplink OFDMA systems," IEEE Transactions on Wireless Communications, vol. 7, no. 5, pp. 1667-1675, May 2008.

[8] M. Maddah-Ali, A. Mobasher, and A. Khandani, "Fairness in multiuser systems with polymatroid capacity region," IEEE Transactions on Information Theory, vol. 55, no. 5, pp. 2128-2138, May 2009.

[9] Z. Han and K. J. R. Liu, Resource Allocation for Wireless Networks. Cambridge University Press, 2008.

[10] W. Yu, W. Rhee, S. Boyd, and J. Cioffi, "Iterative water-filling for gaussian vector multiple-access channels," IEEE Transactions on Information Theory, vol. 50, no. 1, pp. 145-152, Jan. 2004.

[11] K. Kim, Y. Han, and S.-L. Kim, "Joint subcarrier and power allocation in uplink OFDMA systems," IEEE Communications Letters, vol. 9, no. 6 , pp. 526-528, June 2005 . 